\title{
COMPORTAMENTO DO CONSUMIDOR PAULISTA DE PESCADO
}

\author{
Behavior of the Paulista Consumer of Fish
}

\section{RESUMO}

O objetivo do presente trabalho foi analisar o comportamento do consumidor paulista de pescado considerando os aspectos referentes ao hábito de consumo, atributos do produto e fatores sociodemográficos. Realizou-se coletas de dados junto aos consumidores do estado de São Paulo (aproximadamente dois meses), utilizando abordagem online (google docs) e entrevistas presenciais na região metropolitana de São Paulo, resultando em 323 unidades amostrais. Aplicou-se uma regressão de mínimos quadrados ordinários (MQO) e obteve-se os fatores que determinam a frequência média do consumo de pescado. Os resultados evidenciaram que o consumo de pescado ocorre em média 65 vezes ao ano, sendo prioritariamente realizada por homens e mulheres que não possuem crianças e moram sozinhos ou dividem a casa com poucas pessoas. Outro dado observado foi a respeito da necessidade do consumidor por produtos que forneçam maior valor agregado, como o filé de pescado embalado. Além disso, a variável religiosidade (sexta feira santa e natal) mostrou ser um fator determinante na frequência do consumo anual deste produto. Portanto, sugere-se ao setor aquícola e pesqueiro o fornecimento do filé de pescado embalado, explorando os atributos extrínsecos ao produto, a implementação de campanhas de marketing visando ampliar a sua visibilidade do produto, bem como o desenvolvimento de produtos destinado ao público infantil. Todas as estratégias de marketing mencionadas, serão fundamentais para atender as necessidades ao mercado consumidor paulista que busca inovação nos produtos e ainda possui restrições culturais ao consumo.

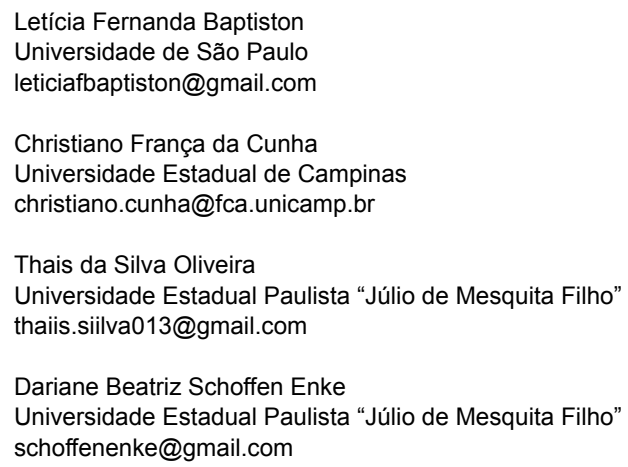

Recebido em: 22/09/2019. Aprovado em: 02/04/2020.

Avaliado pelo sistema double blind review

Avaliador científico: Paulo Henrique Montagnana Vicente Leme

DOI: $10.48142 / 2238-68902019 v 21 n 1-3 p 161172$

\begin{abstract}
The objective of this work was to analyze the behavior of the São Paulo fish consumer, considering the aspects references to the consumption habit, attributes of the product and sociodemographic factors. Data were collected from consumers in the state of São Paulo (approximately two months), using online approach (google docs) and face-to-face interviews in the metropolitan region of São Paulo, resulting in 323 sample units. A regression of ordinary least squares (OLS) was applied and the factors that determine the average frequency of fish consumption were obtained. The results evidenced that the average frequency of fish consumption is 65 times a year, being primarily performed by men and women who do not have children and live alone or divide the house with few people. Another data observed was regarding the consumer's need for products that provide greater added value, such as the packaged fish fillet. In addition, the religiosity variable (Good Friday and Christmas) proved to be a determining factor in the frequency of annual consumption of this product. Therefore, it is suggested to the aquaculture and fisheries sector the supply of packaged fish fillet, exploring the extrinsic attributes to the product, the implementation of marketing campaigns aiming to increase its visibility of the product, as well as the development of products aimed at children. All the mentioned marketing strategies will be essential to meet the needs of the São Paulo consumer market that seeks innovation in products and still has cultural restrictions on consumption.
\end{abstract}

Palavras-chave: Marketing. Segmentação. Recursos Pesqueiros. Frequência do Consumo.

Keywords: Marketing. Segmentation. Fisheries Resources. Frequency of Consumption. 


\section{INTRODUÇÃO}

O pescado (peixes e frutos do mar) são recursos alimentares que possuem todos os nutrientes importantes para a saúde humana, visto seu elevado teor proteico e biológico, com ácidos graxos poli-insaturados, vitaminas e baixo teor de colesterol. Todos esses atributos intrínsecos ao produto, tornam-o mais saudável que outros produtos cárneos (GONÇALVES, 2011; KAWARAZUKA; BÉNÉ, 2010; SARTORI; AMANCIO, 2012; SUN, 2008).

Nos últimos anos, observou-se o aumento da demanda pelo pescado (JOHNSTON; LUND, 2000; MYRLANDA; TRONDSEN, 2000). De acordo com a FAO (Food of Agriculture and Organization of the United Nations, 2018) o consumo de pescado obteve uma taxa de crescimento de 5\% ao ano. $\mathrm{O}$ valor per capita consumido no mundo passou de 9,0 kg em 1961 para aproximadamente 20,3 kg em 2017 e $20,5 \mathrm{~kg}$ em 2018. A mesma organização afirma que durante o ano de 2015, foram produzidas 149 milhões de toneladas de pescado, com destaque para a Ásia com $24 \mathrm{~kg}$ per capita de pescado consumido, seguido pelos países da Oceania com 25 kg per capita, Europa com 22,5 kg per capita e América do Norte 21,6 kg per capita. Nota-se que o menor consumo foi realizado pela África 9,9 kg per capita e a América Latina e o Caribe 9,8 kg per capita.

Segundo a Associação Brasileira da Piscicultura (PEIXE BR, 2019), o consumo de pescado pelos brasileiros gira em torno de $10 \mathrm{~kg} / \mathrm{hab} / \mathrm{ano}$, pouco desenvolvido se comparado a média do consumo mundial de $20 \mathrm{~kg} / \mathrm{hab} /$ ano. Porém de acordo com Sonoda \& Shirota (2012) caso fosse considerado apenas os consumidores que ingerem $o$ pescado, o valor per capita seria de aproximadamente 27,2 $\mathrm{kg}$, visto que há um baixo percentual de consumidores, mas estes consomem quantidades significativas. Segundo Lopes, Oliveira e Ramos (2016) a população brasileira possui preferência de consumo pela carne bovina, seguido de aves e peixes. Estes mesmos autores verificaram que, no norte a preferência pela carne de peixe correspondeu a $70,7 \%$ dos entrevistados em comparação à apenas 15,2\% dos consumidores da região Sudeste. Tavares et al. (2013) argumentam que a frequência de consumo do pescado oscila conforme a proximidade de rios e mares, o que torna este produto mais acessível para os consumidores da região Norte. Esta questão da adjacência aos corpos hídricos também é importante segundo Lopes, Oliveira e Ramos (2016), pois é um fator que eleva o preço do produto, visto questões como distribuição e processamento, tornando-o menos competitivo no mercado para as regiões mais distantes destes locais de origem.
No que diz respeito ao consumo de pescado no estado de São Paulo, um estudo realizado pelo Centro para los Servicios de Información y Asesoramiento sobre la Comercialización de los Produtos Pesqueiros de América Latina y el Caribe (INFOPESCA, 2010), a região metropolitana de São Paulo destaca-se no Brasil como o maior centro consumidor de pescado, visto que dentre todo o volume importado por este país, $49 \%$ era absorvido pelo estado de São Paulo e deste volume estadual $50 \%$ era consumido na região metropolitana de São Paulo. A respeito dos principais produtos e espécies de pescado que eram importados no Brasil, o salmão chileno resfriado, o bacalhau norueguês e o filé de merluza argentino faziam parte do cardápio paulistano, e cerca de $64 \%, 52 \%$ e $49 \%$ do volume total de importações, eram absorvidos pelo estado de São Paulo.

Devido a todos os fatores que foram expostos, o presente artigo tem por objetivo determinar o comportamento do consumidor no estado de São Paulo, visando identificar a frequência de consumo e os fatores que determinam o comportamento de consumo entre os paulistas, e assim, sugerir estratégias de marketing que poderão auxiliar no desenvolvimento dos aspectos mercadológicos dos produtos pesqueiros e aquícolas, que serão fundamentais para ampliar o consumo dentro do estado de São Paulo.

$\mathrm{O}$ artigo foi estruturado da seguinte forma: a partir desta seção introdutória apresenta-se o referencial teórico sobre marketing e a abordagem estratégica aplicada na pesquisa "comportamento do consumidor", em seguida mostra-se os procedimentos metodológicos que foram utilizados para 0 desenvolvimento da pesquisa. Este foi apresentado em três etapas (i) coleta de dados, (ii) definição das hipóteses, (iii) análise dos dados seguido de resultados, discussão e estratégias de marketing. Posteriormente o artigo apresenta a conclusão com as considerações finais e as referências bibliográficas. Enfatiza-se a relevância deste artigo, visto que durante a revisão bibliográfica notou-se certa escassez de informações a respeito dos consumidores de pescado no estado de São Paulo.

\section{REFERENCIAL TEÓRICO}

O marketing é um processo administrativo e social, em que indivíduos e organizações obtêm o que necessitam e desejam pela criação e troca de valor com seus clientes. Portanto, o objetivo do marketing é promover a satisfação dos clientes, que ocorre quando as organizações fornecem produtos e serviços com valor superior ao cliente (KOTLER; ARMSTRONG, 2015).

Por definição, consumidor é o indivíduo que possui o desejo ou uma necessidade por algo (SOLOMON, 2016).

Organizações Rurais \& Agroindustriais, Lavras, v. 21, n.1-3, p. 161-172, 2019 
O processo de motivação e emoção dos consumidores determinam a compra e deve ser avaliado continuamente (HAWKINS; MOTHERSBAUGH; BEST, 2007), visto que, o consumidor no momento da compra avalia os benefícios (atendimento, variedade e conforto) e os custos (preço dos produtos e condições de pagamento). Essa relação (custo e benefício) determinarão as preferências dos consumidores (PARENTE, 2010), ou seja, o comportamento do consumidor.

Trata-se de um tema interdisciplinar que envolve o conhecimento de economia, sociologia, antropologia cultural, semiótica, demografia e história, sendo de extrema relevância para identificar o público-alvo (PINHEIRO et al., 2015).

Após a compreensão dos consumidores, do mercado e do público-alvo, elaboram-se as estratégias de marketing orientadas para o cliente (KOTLER; ARMSTRONG, 2015). Dessa forma, é possível fornecer a proposta de valor, visando manter os consumidores por meio de um conjunto de benefícios que irão suprir suas necessidades, gerar satisfação e, assim, manter seus clientes (SCHARF, 2012).

Estudos sobre o comportamento do consumidor de pescado foram realizados na Europa e na Ásia. Leek, Maddock e Foxall (2000) verificaram que o consumo de pescado era baixo devido a percepção do consumidor sobre o produto, pois este acredita tratar-se de um produto de difícil aquisição, sendo necessário investir tempo na compra e no preparo. Estes mesmos autores relataram que o pescado mostrou-seum produto inacessível economicamente, outro aspecto negativo observado pelos consumidores foi a presença de ossos e o odor característico do produto, que muitas vezes não são aceitos pelos consumidores de produtos cárneos.

Verbeke e Vackier (2005) estudaram o comportamento planejado do consumidor de pescado na Bélgica e verificaram que a frequência do consumo foi inferior ao recomendado pelas autoridades locais (duas vezes por semana). Além disso, os autores observaram que os fatores que determinam a intenção de consumo de peixes, são a pressão social dos colegas, a responsabilidade moral e a convicção em possuir habilidades para a escolha e o preparo do produto nos lares familiares.

Kaimakoudi et al. (2013) estudaram o comportamento dos consumidores gregos em relação ao pescado proveniente da pesca extrativista e o potencial de consumo do pescado aquícola. De acordo com os autores, os consumidores que possuíam potencial de consumo do pescado aquícola era composto por indivíduos que possuiam um elevado grau de instrução. Os mesmos autores afirmaram ainda, que para elevar a demanda pelo pescado aquícola e o valor deste produto no mercado Grego seria necessário incentivar a conscientização dos consumidores sobre o produto, realizar a implementação de estratégias de marketing mais eficientes e investir em diferentes formas de promoção do produto.
Carlucci et al. (2015) revisaram o padrão de consumo de compra de pescado nos países desenvolvidos. Estes autores verificaram que o consumo deste produto é realizado principalmente por indivíduos que possuem atividade de consumo regular, motivados principalmente pelos aspectos de saudabilidade conferido pela ingestão do produto. Quanto as barreiras de consumo do pescado, os autores observaram que os atributos intrínsecos (odor) e extrínsecos (produtos de baixa qualidade e preços elevados) reduzem a frequência do consumo.

Khan, Aldosari e Hussain (2016) estudaram o comportamento do consumidor de pescado no Reino da Arábia Saudita e verificaram que o consumo de pescado ocorre aproximadamente duas vezes por semana, realizado majoritariamente por indivíduos com idades entre 25 a 34 anos, que possuem ensino superior e preferem ingerir peixes ao invés de outros produtos cárneos, devido aos fatores nutricionais do pescado.

É possível observar que o consumo de pescado elevou-se no mundo todo, portanto é necessário estudar os consumidores para assim compreender o que eles pensam sobre o produto, a forma de consumo realizada, quantidade que comprarão a cada vez, frequência do uso, hábitos de compra em relação ao local e quais desejos serão satisfeitos com o uso deste produto (MAZZUCHETTI; BATALHA, 2005). Compreender o consumidor é imprescíndivel para realizar estratégias de marketing eficazes, e assim, ampliar o consumo e a exportação de peixes (SUPARTINI; OISHI; YAGI, 2018). Diversos estudos de marketing foram realizados na Europa e em alguns países asiáticos visando compreender os fatores que afetam a decisão de compra e o consumo de pescado.

Se for considerado o grupo de produtos carnes, aves e pescado, uma pesquisa publicada pelo Instituto Brasileiro de Geográfia e Estatística (IBGE, 2019) mostrou que o Sudeste possui despesas médias mensais de $18,1 \%$, enquanto que o Norte do país apresenta a maior porcentagem de despesas para este grupo de alimentos $(27,1 \%)$, porém não é possível identificar especificamente quanto dessa porcentagem de despesa média deve-se ao consumo de pescado.

Notou-se certa escassez de informações sobre os compradores e consumidores de pescado no Brasil (SOUZA, 2002), porém diversos estudos apontam que o consumo de pescado ocorre principalmente na região norte e nordeste do país, mas que conforme apresentado acima, o estado de São Paulo no ano 2010, foi caracterizado como um grande centro consumidor do país, realizado principalmente pela Região Metropolitana de São Paulo (RMSP), com uma média de 15,1 kg/habitantes/ano, abastecida principalmente pelo pescado importado e pela pesca extrativista que ocorre em seu litoral norte e sul (INFOPESCA, 2010). 


\section{METODOLOGIA}

\subsection{Coleta de Dados}

As seguintes etapas foram necessárias para esta coleta de dados: a elaboração dos questionários, a divulgação online destes e as coletas dos dados. Para realizar a coleta dos dados, elaborou-se um formulário online no google forms. No período inicial da coleta, entrou-se em contato com revistas técnicas do setor aquícola e pesqueiro que divulgaram a pesquisa e o link dos questionários aos seus clientes por e-mail. Realizou-se também a divulgação destes links através de outras mídias-sociais, como instagram e facebook, sendo que essas duas formas de divulgação dos questionários foram as principais estratégias utilizadas durante todo o período de coleta dos dados (25 de outubro a 27 de novembro de 2018). Visando ainda obter uma amostra mais significativa na capital e na região metropolitana de São Paulo, realizou-se entrevistas presenciais junto aos clientes de feiras e mercados municipais por um período de duas semanas.

As entrevistas ocorreram primeiramente na cidade de Cotia, que é um polo de desenvolvimento da Região Metropolitana de São Paulo e que possui quatro macrorregiões ao longo da Rodovia Raposo Tavares. A região detém o parque industrial da cidade de Cotia, assim como a cidade de Granja Viana situada no polo e que possui centros gastronômicos. Devido a essas características, a cidade de Cotia recebe grande fluxo de turistas de negócios e clientes (habitantes da região) que frequentam o polo e realizam compras de alimentos nos supermercados, feiras e mercado municipal, sendo este o motivo da escolha das entrevistas neste município (PREFEITURA DE COTIA, 2019).

Foram realizadas entrevistas na cidade de São Paulo (capital) no "Mercado Municipal de São Paulo" e nas feiras livres localizadas no bairro "Novo Brooklin", por ser um bairro onde situam-se grandes centros empresariais, com elevado fluxo de pessoas e que possui feiras livres todos os dias (SÃO PAULO ANTIGA, 2019).

Por meio das coletas (online e entrevistas presenciais), obteve-se uma amostra composta por 323 entrevistados, respectivamente, 151 consumidores do interior do estado, 106 na capital, 26 no litoral de São Paulo e 40 consumidores domiciliados na região metropolitana de São Paulo.

O questionário foi elaborado de modo a obter dados socioeconômicos, motivacionais e as percepções sobre os atributos do produto, e como esses atributos impactam o consumo de pescado pelos entrevistados, para isso adaptouse o questionário de Vasconcelos (2010) aplicado aos compradores de pescados em feiras no município de Santo
André, e o estudo sobre o comportamento do consumidor de carne bovina realizado por Souki, Salazar, Antonialli e Pereira (2003), que estudaram os atributos determinantes na decisão de compra.

Segundo Malhotra (2001) as pesquisas que possuem dentro do banco de dados fatores qualitativos e quantitativos geram resultados eficazes e garantem a implementação de estratégias de marketing precisas. De acordo com Verbeke e Vackier (2005) estudos sobre o hábito e comportamento de consumo, devem abordar os fatores que influenciam a escolha do produto, e assim obter uma descrição completa na relação entre o consumidor e um determinado produto. Portanto, na presente pesquisa o questionário aplicado aos consumidores de pescado foi elaborado de modo a compor informações sobre a qualidade do produto, dados socioeconômicos, atitudinais e motivacionais dos consumidores, sobre a decisão de consumir pescado, e assim, aferir a frequência média de consumo de pescado realizada pelos paulistas.

Para isso, a primeira parte do questionário abordou questões individuais do consumidor (gênero, idade, cidade de residência, número de pessoas na residência, possuir ou não criança na residência, profissão e escolaridade). A segunda parte abordou o hábito de consumo de pescado (comer ou não pescado, motivo do consumo, frequência do consumo, local de compra, quais tipos de pescado consome, preferência na embalagem do produto, influências e motivos do consumo atual, e motivos para aumentar o consumo individual e da população). A penúltima questão referiu-se às afirmativas sobre os atributos extrínsecos/intrínsecos do pescado (preço, sabor, rastreabilidade, rápida deterioração e presença de espinhas), e foi respondida em uma escala Likert de cinco pontos. A última questão foi a respeito da renda mensal (em salários mínimos) dos consumidores.

\subsection{Definição das Hipóteses}

Por meio de análise bibliográfica, determinou-se as seguintes hipóteses:

1. $\mathrm{H}_{1}$ A frequência do consumo de pescado é superior na região metropolitana de São Paulo, visto ser considerado um grande centro consumidor no estado (INFOPESCA, 2010);

2. $\mathrm{H}_{2}$ : A frequência do consumo de pescado aumentará com o fornecimento de pescado (posta ou filé embalado), tornando o produto de fácil preparo (mais conveniência ao consumidor) e maior valor agregado ao produto (LOPES; OLIVEIRA; RAMOS, 2016; NIELSEN; SØARENSU; GRUNET, 1997 apud GRUNET, 2005; OLSEN, 2003; SOUZA, 2002); 
3. $\mathrm{H}_{3}$ A frequência do consumo de pescado é altamente influenciada pelo preço, ou seja, quanto maior o seu preço, menor o seu consumo (CARLUCCI et al., 2015; FIGUEIRO; SOUSA; CASTRO, 2014);

4. $\mathrm{H}_{4}$ : A frequência do consumo é menor quanto maior o número de pessoas nos domicílios (VASCONCELOS, 2010);

5. $\mathrm{H}_{5}$ : A frequência do consumo de pescado em domicílios que possuem crianças será inferior, possivelmente devido a presença de espinhos (BORTOLINI; GUBERT; SANTOS, 2012; LEEK; MADDOCK; FOXALL, 2000; VERBEKE; VACKIER, 2005,);

6. $\mathrm{H}_{6}$ : Pesquisas sobre o comportamento de compra e consumo de pescado demonstram que a idade dos consumidores possui relação direta na determinação de compra e aumento do consumo, visto que com o acréscimo na idade as pessoas se preocupam mais em ingerir alimentos saudáveis (BRUNSØ et al. 2009; KHAN; ALDOSARI; HUSSAIN, 2016; MYRLAND et al. 2000; OLSEN, 2003);

7. $\mathrm{H}_{7}$ : Fatores sociais e culturais como a religiosidade influenciam positivamente na frequência do consumo de pescado (LOPES; OLIVEIRA; RAMOS, 2016; ROCHA et al. 2013; SUPARTINI; OISHI; YAGI, 2018).

\subsection{Análise dos Dados}

Todas as variáveis independentes foram codificadas e submetidas a regressão multivariada pelo método de mínimos quadrados ordinários (MQO). Este método é uma estratégia eficiente para estimar os parâmetros da regressão. Utilizou-se o software Stata (Software for Statistics and data Science) para realizar a análise estatística dos dados. A variável dependente utilizada foi a frequência anual do consumo de pescado (número de dias anuais), e como variáveis independentes foram utilizadas: o gênero; a idade; a região de residência; o número de pessoas em cada residência; presença de criança na residência; o nível de escolaridade; se consome ou não pescado; os motivos do consumo; o local de compra; os motivos que aumentariam o consumo de pescado pelo entrevistado; os motivos que aumentariam o consumo da população; os possíveis fatores determinantes do consumo considerando as medidas de concordância do entrevistado, por meio de escala Likert, sobre os diversos atributos extrínsecos e/ou intrínsecos (preço, sabor, rastreabilidade, rápida deterioração, presença de espinhos); e a renda mensal dos entrevistados, em salários mínimos.

\section{RESULTADOS}

Na Tabela 1 é possível observar alguns componentes socioeconômicos dos 323 consumidores que colaboraram com a pesquisa. Nota-se que $63 \%$ destes consumidores são do sexo feminino e $37 \%$ do sexo masculino. A abordagem utilizada nesta pesquisa (coletas online e entrevistas presenciais) podem ter colaborado com esse resultado. Minozzo, Haracemiv e Waszczynskji (2008) e Vasconcelos (2010) estudaram o perfil do consumidor de pescado no município de São Paulo e no município de Santo André (RMSP), respectivamente, e observaram que as mulheres demonstraram ser mais atenciosas e interessadas em colaborar com a pesquisa. Minozzo, Haracemiv e Waszczynskji (2008) afirmam que as mulheres costumam ser responsáveis por realizar as compras de alimentos nas famílias, o que também colabora com o dado obtido e Verbeke e Vackier (2005) verificaram que as mulheres consomem mais pescados, por se preocuparem mais com os aspectos de saúde se comparado com os homens.

Quanto a idade, observa-se que os consumidores que participaram desta pesquisa possuem em média 34 anos de idade (variando entre 18 a 80 anos), e 40\% destes consumidores possuem idade igual ou inferior a 25 anos, com renda mensal entre 1 à 3 salários mínimos $(31,9 \%)$, com escolaridade incompleta $(34,0 \%)$ e completa $(26,0 \%)$. Por meio dos dados coletados, nota-se que os consumidores que participaram desta pesquisa compõe em sua maioria jovens.

Na presente pesquisa, os resultados obtidos podem ter sido influenciados pelo método de coleta de dados que foi aplicado (questionário online e entrevistas realizadas no período matutino e vespertino), visto que Minozzo, Haracemiv e Waszczynskji (2008) e Vasconcelos (2010) afirmam que quando o método de coleta ocorre em períodos matutino e vespertino e coletas online é possível obter uma amostra de consumidores mais heterogêneos.

A análise do presente estudo foi estruturada de modo a obter a frequência média do consumo (anual) de pescados realizada pelos consumidores residentes no estado de São Paulo. Observa-se que a estimativa do modelo total está apresentada na Tabela 2 . O valor de $\mathrm{R}^{2}$ foi de 0,16 , um valor considerado bom para as pesquisas sociais (HAIR et al. 2005) e próximo do obtido por Olsen (2003) em seu estudo sobre os consumidores de pescado na Noruega. Os resultados apresentados em vermelho e em negrito nesta tabela são as variáveis significativas que possuem influências diretas na equação de frequência do consumo de pescado (Equação 1). Esta equação expõe a influência das seguintes variáveis: a) Número de pessoas na casa (NPC), b) presença de criança na residência (PC); c) consumo motivado por aspectos de religiosidade (MR) e; d) preferência de compra do filé-embalado (PFE). 
TABELA 1 - Características Sócio-demográficas dos consumidores, em porcentagem

\begin{tabular}{|c|c|c|c|c|c|}
\hline \multirow{2}{*}{ Gênero } & Masculino & 36,8 & \multirow{2}{*}{ Criança na residência } & $\mathrm{Sim}$ & 27,2 \\
\hline & Feminino & 63,1 & & Não & 72,8 \\
\hline \multirow{5}{*}{ Idade } & $\leq 25$ anos & 40,5 & \multirow{6}{*}{ Renda mensal } & $\leq \mathrm{R} \$ 954,00$ & 21,4 \\
\hline & 26-35 anos & 19,8 & & $\mathrm{R} \$ 954-\mathrm{R} \$ 2.862,00$ & 31,9 \\
\hline & $36-45$ anos & 14,5 & & $\mathrm{R} \$ 2.862,00-\mathrm{R} \$ 4.770,00$ & 22,3 \\
\hline & 46-55 anos & 14,8 & & $\mathrm{R} \$ 4.770,00-\mathrm{R} \$ 9.540,00$ & 14,2 \\
\hline & $>55$ anos & 10,4 & & $\mathrm{R} \$ 9.540,00-\mathrm{R} \$ 14.310,00$ & 7,8 \\
\hline \multirow{5}{*}{$\begin{array}{l}\text { Região de } \\
\text { residência }\end{array}$} & Média (idade) & 34 & & $>\mathrm{R} \$ 14.310,00$ & 2,4 \\
\hline & Capital & 32,8 & & & \\
\hline & Região metropolitana & 12,4 & \multirow{8}{*}{ Escolaridade } & Sem estudo & 0 \\
\hline & Litoral & 8,0 & & Fundamental incompleto & 2,1 \\
\hline & Interior & 46,7 & & Fundamental completo & 1,2 \\
\hline \multirow{5}{*}{$\begin{array}{c}\mathrm{N}^{\mathrm{o}} \text {. de } \\
\text { moradores }\end{array}$} & & & & Médio incompleto & 1,5 \\
\hline & 1 a 2 pessoas & 34 & & Médio completo & 18,2 \\
\hline & 3 ou 4 pessoas & 52 & & Superior incompleto & 34 \\
\hline & 5 ou mais pessoas & 14 & & Superior completo & 26 \\
\hline & & & & Pós-graduação & 16,71 \\
\hline
\end{tabular}

Fonte: Dados coletados na pesquisa

Portanto, observa-se abaixo a equação de frequência do consumo (anual) com as variáveis que determinam o comportamento do consumo:

$F C=65,4^{*}-5,55\left(N P C^{*}\right)-11,78\left(P C^{* *}\right)-$

$-40,94\left(M R^{* *}\right)+10,48(P F E)$

Onde: ${ }^{*}=$ significante ao nível de $10 \% \mathrm{e}^{* *}=$ significante ao nível de $5 \%$

\section{DISCUSSÃO}

Conforme apresentado na equação 1, a frequência média do consumo de pescado dos paulistas é de 65,41 vezes ao ano, sendo este consumo influenciado pelos fatores descritos abaixo.

Quanto a hipótese 1, essa foi rejeitada ao nível de $10 \%$, pois o coeficiente da variável dummy "cidade de residência (Região metropolitana de São Paulo)" na Tabela 1 não foi significativo a tal valor. Ou seja, de acordo com os resultados obtidos nessa pesquisa, o consumo de pescado ocorre igualmente nas regiões pesquisadas, não havendo assim uma concentração de consumo de pescado na capital do estado como previsto pelo Infopesca (2010). Esta ausência de diferença pode ter ocorrido, pois a pesquisa deste autor foi realizada há mais de nove anos e esta situação de concentração pode ter sido minimizada, com uma maior ampliação deste consumo nas demais regiões do estado.

No caso da hipótese 2, conforme apresentado no resultado da regressão, o comprador de pescado no estado de São Paulo possui preferência em adquirir o filé-embalado, pois o seu coeficiente teve significância ao nível de 5\%. Portanto, os produtos que conferirem estes atributos aos consumidores possuirão um acréscimo de $16,02 \%$ no consumo de pescado (10,48 vezes ao ano). Desta forma, não se pode rejeitar que a frequência do consumo de pescado aumentará com o fornecimento de um produto que possua uma apresentação mais elaborada (filé-embalado). Trata-se de um atributo que representa a conveniência ao consumidor, visto que este busca por produtos que confiram maior facilidade na aquisição e preparo do produto. Estes fatores são importantes para ampliar o consumo entre as pessoas que possuem o hábito de consumir pescado, e ainda pode ser estratégico para adquirir novos clientes que não estão acostumados a ingerir o produto. Esta constatação está em conformidade com Leek, Maddock e Foxall (2000), Arvanitoyannis et al. (2004) e Birch e Lawley (2012), que verificaram em suas pesquisas que alguns consumidores consideram a embalagem um fator de conveniência e de confiabilidade na qualidade e no rastreamento do produto. Segundo Barbosa et al. (2006), os atributos externos ao pescado são avaliados pelos consumidores no momento da compra. 
TABELA 2 - Estimativa do modelo total

\begin{tabular}{|c|c|c|}
\hline Variável & Coeficientes & Desvio Padrão \\
\hline Gênero & $-6,742$ & $(4,547)$ \\
\hline Cidade de residência (Interior de São Paulo) & 3,066 & $(5,248)$ \\
\hline Cidade de residência (Litoral de São Paulo) & 11,07 & $(8,723)$ \\
\hline Cidade de residência (Região metropolitana de São Paulo) & 4,884 & $(7,106)$ \\
\hline Quantas pessoas residem em sua casa & $-5,551 *$ & $(3,347)$ \\
\hline Presença de criança em casa & $-11,78 * *$ & $(5,208)$ \\
\hline Qual sua escolaridade & 1,990 & $(1,920)$ \\
\hline o/a senhor(a) come peixe/camaro/ostras & $-13,53$ & $(25,13)$ \\
\hline Motivos (Paladar) & 2,909 & $(4,962)$ \\
\hline Motivos (Hábito) & 22,06 & $(15,86)$ \\
\hline Motivos (Religiosidade) & $-40,95 * *$ & $(17,43)$ \\
\hline Motivos (Preferência por pescado) & $-20,02$ & $(13,17)$ \\
\hline Motivos (Variação do cardápio) & 10,94 & $(11,90)$ \\
\hline Motivos (Cultura familiar) & 2,111 & $(15,74)$ \\
\hline Motivos (Não informou) & $-11,65$ & $(18,79)$ \\
\hline Motivos (Vontade) & $-20,12$ & $(23,79)$ \\
\hline Motivos (Quase não consome) & $-15,69$ & $(21,69)$ \\
\hline Motivos (Outros) & $-19,48$ & $(22,66)$ \\
\hline Local de compra (Supermercado) & $-2,580$ & $(5,276)$ \\
\hline Local de compra (Restaurante) & $-8,593$ & $(6,183)$ \\
\hline Local de compra (Pescador) & $-1,481$ & $(7,877)$ \\
\hline Preferência no momento da compra (Enlatado) & 4,494 & $(13,77)$ \\
\hline Preferência no momento da compra (Filé-embalado) & $10,48^{*}$ & $(5,969)$ \\
\hline Preferência no momento da compra (Posta embalada) & 9,669 & $(8,823)$ \\
\hline Cite os motivos que o faria consumir mais (Qualidade superior) & 8,584 & $(14,82)$ \\
\hline Cite os motivos que o faria consumir mais (Disponibilidade de produto fresco) & $-10,46$ & $(11,74)$ \\
\hline Cite os motivos que o faria consumir mais (Menor preço) & $-2,039$ & $(4,705)$ \\
\hline Cite os motivos que o faria consumir mais (Produtos mais acessíveis) & $-9,818$ & $(8,484)$ \\
\hline Cite os motivos que o faria consumir mais (Outros) & $-21,79$ & $(21,76)$ \\
\hline Cite os motivos que o faria consumir mais (Praticidade) & $-17,39$ & $(12,99)$ \\
\hline Preço & 1,840 & $(1,625)$ \\
\hline Sabor & 2,838 & $(2,470)$ \\
\hline Rastreabilidade & $-1,366$ & $(1,758)$ \\
\hline Rapida deterioração & 2,057 & $(1,674)$ \\
\hline Presença de espinhos & $-1,407$ & $(1,652)$ \\
\hline Renda & 2,255 & $(1,933)$ \\
\hline Constante & $65,41 * *$ & $(31,68)$ \\
\hline Observações & 323 & \\
\hline $\mathrm{R} 2$ & 0,160 & \\
\hline
\end{tabular}

Fonte: Dados da pesquisa 
Em relação a hipótese 3 nota-se que esta foi rejeitada por esta pesquisa, pois o coeficiente de preço na Tabela 2 não foi significativo ao nível de $10 \%$, o que indica que este fator não influência a frequência do consumo. Assim como na presente pesquisa, alguns autores afirmaram que os consumidores identificam que o preço do pescado é um fator negativo, mas que não afeta a frequência do consumo (LEEK; MADDOCK; FOXALL, 2000; LOPES; OLIVEIRA; RAMOS, 2016; VERBEKE; VACKIER, 2005 ,). Esse resultado pode ser explicado pelo perfil dos entrevistados, composto por pessoas com 35 anos em média e com bom nível de escolaridade, variáveis que aumentam a frequência do consumo de pescado (KAIMAKOUDI et al. 2013; VERBEKE; VACKIER, 2005). Nota-se também, que os entrevistados possuem maior preocupação com a agregação de valor no pescado, pois desejam produtos como o filé de pescado, conforme observado na hipótese 2, o que faz com que o preço não seja o fator determinante para o consumo. Pode-se ainda enfatizar este resultado utilizando os dados publicados pelo IBGE (2019), o qual mostrou que, dentre todas as regiões estudadas, o Sudeste possui o segundo maior percentual de consumidores que se alimentam fora dos domicílios $(34,2 \%)$. Quando considerado o grupos de alimentos preparados, a região Sudeste apresentou o maior percentual de despesa média mensal $(4,1 \%)$, resultados estes que sugerem que os consumidores que residem no Sudeste buscam alimentos que confiram facilidade no preparo e consumo de alimentos.

No que concerne a hipótese 4 , observa-se que a cada mudança na faixa do número de pessoas na residência, por exemplo da faixa de 1-2 pessoas para 3-4 indivíduos, haverá uma diminuição de $8,48 \%$ no consumo anual ( 5,55 vezes ao ano), passando a ser 59,86 vezes ao ano. Por meio desse resultado, não se rejeita ao nível de $10 \%$ a hipótese de que o número de pessoas na residência altera a frequência média do consumo. Segundo o IBGE (2019), na região Sudeste as despesas médias mensais considerando o grupo de alimentos carnes, aves e pescado é a segunda menor do país (18,1\%), com uma média de 2,9 pessoas por grupo familiar. Esses dados também foram verificados pelo Infopesca (2010), que observaram que indivíduos que moram sozinhos e casais sem filhos representam os grupos de consumidores que, possivelmente, mais frequentam restaurantes e fast-foods que comercializam pescado na cidade de São Paulo.

Quanto a hipótese 5 nota-se que a variável "presença de criança na residência" reduz a frequência do consumo de pescado, portanto, o consumidor que residir com crianças e/ou possuírem filhos reduzirão o consumo de pescado em $18 \%$ (11,78 vezes ao ano), ou seja, o consumo será de 53,63 vezes ao ano. Portanto, não se pode rejeitar ao nível de $5 \%$ esta hipótese de que a frequência do consumo diminui em residências que possuem crianças. Leek, Maddock e Foxall (2000) aferiram que nos domicílios que possuem crianças, o consumo de pescado é possivelmente menor devido a presença de espinhos na carne, resultando no receio dos pais em inserir o peixe na alimentação dos filhos. Conforme observado por alguns autores, essa redução de consumo de pescado também esta relacionada com um fator caracterizado como "pressão social". Isso comumente ocorre quando um ou mais individuos não consomem determinado produto dentro do grupo familiar, neste caso, os responsáveis por realizar a compra optam por outros produtos, fato este que costuma ocorrer entre os jovens, visto que o consumo de pescado é menor entre esse grupo de consumidores, o que reduz a frequência do consumo (VERBEKE; VACKIER, 2005). Segundo Sartori e Amancio (2012), o consumo de pescado entre crianças e adolescentes é classificado como baixo, devido ao sabor dos peixes. Os resultados também estão em consonância com Bortolini, Gubert e Santos (2012), que constataram que somente $35,9 \%$ das crianças da região Sudeste possuíam o hábito de consumo de peixe, caracterizado por, em média, duas vezes por semana. Tais resultados evidenciam que as crianças no estado de São Paulo não possuem o hábito de consumir pescado.

No que diz respeito a hipótese 6 esta foi rejeitada. Estudos realizados na Europa demonstram que a idade possui relação direta com o consumo de pescado, visto que conforme o acréscimo na idade do consumidor, este preocupa-se mais com a saúde, passando a consumir alimentos mais saudáveis (OLSEN, 2003; SUPARTINI; OISHI; YAGI, 2018; VERBEKE; VACKIER, 2005).

Com relação a hipótese 7, nota-se que a categoria "religiosidade", que é um fator cultural e motivacional foi relevante e pertinente para este consumo, pois o seu coeficiente foi significativo ao nível de 5\%. Muitos consumidores durante as entrevistas relataram realizar a compra e o consumo de pescado devido a suas crenças religiosas e, principalmente durante na sexta-feira santa e o natal. O resultado obtido nessa pesquisa é facilmente explicado, pois o Brasil é um país culturalmente católico, onde ocorre o consumo de bacalhau e outros peixes em datas comemorativas consideradas importantes no cristianismo. Os dados obtidos na presente pesquisa estão em conformidade Rocha et al. (2013), que afirmam que o consumo de pescado pode elevar-se devido as questões religiosas, resultado similar ao observado por Supartini et al. (2018), visto que identificaram que o

Organizações Rurais \& Agroindustriais, Lavras, v. 21, n.1-3, p. 161-172, 2019 
desejo de comprar e consumir pescado no Reino Unido é fortemente relacionado ao fator religioso. Porém, notase que os eventos citados acima, no caso do Brasil, são considerados isolados, mas significativos para a frequência do consumo, visto que a semana santa corresponde a $30 \%$ e o natal a $15 \%$ do volume de pescado consumido no país, época em que a demanda pelo produto se eleva, e os produtores e as processadoras intensificam o trabalho para atender ao mercado nacional (Empresa de Assistência Técnica e Extensão Rural [EMATER], 2018). Portanto, a religiosidade é um fator relevante para o consumo, como pode ser observado na Equação 1, visto que na ausência dos eventos religiosos ocorrerá um decréscimo de $62 \%$ na frequência do consumo anual de pescado (40,94 vezes ao ano), resultando em uma frequência média de consumo de 24,47 vezes ao ano, ou seja, um pouco maior que duas vezes por mês (24 vezes).

\section{ESTRATÉGIAS DE MARKETING}

A partir dos resultados estatísticos obtidos no presente trabalho, pode-se segmentar os consumidores de pescado. Portanto, o público-alvo do pescado no estado de São Paulo é composto, prioritariamente, por homens e mulheres que residem sozinhos ou dividem a residência com poucas pessoas; que não possuem crianças; que realizam o consumo motivados por aspectos religiosos; e que esperam um produto com atributos que confiram maior conveniência na compra e no preparo do produto, resultando em maior valor agregado.

Quanto às famílias que possuem crianças, a hipótese é que o consumo é inferior devido a presença de espinhos na carne do pescado e o forte odor que o produto possui. Portanto, sugere-se a comercialização de produtos congelados, visto que o filé congelado passa por processos de remoção das espinhas, o que reduz o perigo de uma criança sofrer algum acidente, e ainda agrega valor superior ao produto. Estes produtos possuem odor suave se comparado ao pescado fresco, principal barreira do consumo entre as crianças (LEEK; MADDOCK; FOXALL, 2000). Outra alternativa é investir na formulação de produtos utilizando a carne mecanicamente separada (CMS), um subproduto da indústria de filetagem que dispõe de alto teor protéico. Segundo Lopes, Oliveira e Ramos (2016), é fundamental investir na formulação de novos produtos processados para ampliar o consumo de peixes pela população brasileira.

Quanto ao atributo "sem espinho", este deve ser amplamente divulgado na embalagem e/ou outros meios de comunicação para os consumidores, reforçando a ausência deste atributo no produto, visto ser intrínseco ao pescado e somente pode ser percebido após o seu consumo, ou seja, é um bem de experiência (CUNHA; SPERS; ZYLBERSZTAJN, 2011). Verbeke e Vackier (2005) observaram que consumidores que tiveram boas experiências com o pescado não se preocupavam com o fato do produto possuir espinhas e ser visto como um alimento de alto preço.

A respeito do aspecto religioso, é necessário que as empresas do setor aquícola e pesqueiro, como, empresários da aquicultura, processadoras de pescado e organizações envolvidas no setor, implementem estratégias de promoção deste produto em diferentes canais durante o ano e principalmente depois que as festas religiosas ocorrerem, e assim, ampliar o consumo durante o ano todo e não apenas nessas datas que ocorrem de forma isolada.

Quanto ao último requisito apresentado, o marketing pode atuar de forma mais pontual e específica, pois estes consumidores buscam por maior oferta do pescado comercializado em filé-embalado nos estabelecimentos de comercialização do produto. Portanto ao fornecer produtos que atendam as necessidades dos consumidores ocorrerá um aumento do consumo anual em 16\%. Segundo Kaimakoudi et al. (2013) e Lopes, Oliveira e Ramos (2016), estratégias de marketing mais eficientes são fundamentais para promover os produtos oriundos da aquicultura e da pesca, podendo assim contribuir com o desenvolvimento do setor.

Sugere-se o investimento em embalagens que forneçam atributos que confiram ao consumidor versatilidade e funcionalidade, o que é possível por meio de embalagens de diversos tamanhos e formatos, dessa forma, será possível atender as necessidades e desejos do consumidor, e ampliar a construção de marca. Becker (2000) afirma que as características extrínsecas (preço e embalagem) ao produto são uma forma de comunicar os atributos de confiança ao consumidor. Pode-se ainda, trabalhar a embalagem do produto visando informar atributos que são importantes para o consumidor (informações nutricionais e outras características) que podem ampliar o consumo do produto, pois Antonielli, Rezende e Carneiro (2018) afirmam que os estímulos externos, como o rótulo, embalagens e fatores sociais afetam a escolha do produto pelos consumidores.

Outra estratégia que pode ser realizada pelas empresas de pescado envolve a demonstração do produto em mercados, visto que isso contribui para que os consumidores conheçam os produtos, gerando assim, a experiência do produto na memória do comprador, 
ampliando o consumo individual destes clientes. Sugere-se também, a implementação do mix de promoção de vendas, por meio da realização de eventos visando a divulgação do produto, que pode ser realizado pelo setor aquícola e pesqueiro (LOPES; OLIVEIRA; RAMOS, 2016) e por empresas que possuem marcas famosas no mercado, gerando a conexão entre empresa-consumidor. Além disso, seria interessante ampliar os anúncios a respeito deste produto, tornando-o mais visível para todos os consumidores de diversos estados, visto ser uma estratégia pouco utilizada se comparada a outros produtos cárneos comercializados no mercado nacional.

\section{CONCLUSÕES}

O consumo de pescado mundialmente ocorre atrelado aos seus aspectos nutricionais, ao sabor do produto e em função da idade do consumidor. O estudo do comportamento do consumidor é imprescindível para a implementação de estratégias de marketing que podem ampliar a demanda pelo pescado e seus produtos, contribuindo assim com o desenvolvimento do setor aquícola e pesqueiro.

$\mathrm{Na}$ presente pesquisa, observou-se que o comportamento do consumidor de pescado está relacionado com os fatores sociais (possuir filhos e o número de pessoas na residência), motivacionais e culturais (religiosidade) e as características extrínsecas ao produto (embalagem). Este resultado evidência que o público-alvo consumidor de pescado é composto por jovens que não possuem filhos, que realizam o consumo motivados pela religiosidade e que se preocupam com a forma em que o produto está exposto no varejo (filé-embalado). Dessa forma, a indústria de pescado pode investir no desenvolvimento de produtos que confiram maior conveniência ao consumidor na aquisição e no preparo do produto, por meio de embalagens que transmitam essa mensagem.

Pode-se ainda investir no desenvolvimento de novas pesquisas de mercado, visando identificar quais são as barreiras do consumo para as famílias que possuem filhos, e assim, desenvolver produtos direcionados a esse público.

No que diz respeito ao fator religiosidade, este mostrou-se relevante para o consumo anual, portanto, sugere-se a implementação de campanhas de marketing de forma a ampliar a conscientização dos benefícios nutricionais que o pescado fornece, e assim, o consumo de pescado não será advindo somente por este fator cultural que apesar de ser fundamental para as vendas, ocorre primordialmente na semana santa, e trata-se de um evento isolado se considerado o consumo anual.

A realização deste trabalho tentou reduzir a escassez de informações socioeconômicas e comportamentais dos consumidores de pescado no estado de São Paulo, mas ainda observa-se a necessidade de outras pesquisas de marketing junto aos consumidores dos produtos aquícolas e pesqueiros. Portanto, sugere-se o desenvolvimento de novas pesquisas de marketing focadas no consumidor de pescado em outras regiões do país, seu estilo de vida, os atributos intrínsecos e extrínsecos ao produto, situação de utilização do produto e como são transformados em alimentos. Dessa forma, torna-se possível compreender os fatores que determinam a compra do produto, a previsão de demanda futura, o desenvolvimento de estratégias de marketing, a elaboração de novas linhas de produtos e gerar vantagem competitiva ao setor.

\section{REFERÊNCIAS}

ANTONIALLI, F.; REZENDE, D. C.; CARNEIRO, J. D. S. New products development: A marketing study of a popsicle produced with whey. Organizações Rurais \& Agroindustriais, Lavras, v. 20, n. 1, p. 1-14, 2018.

ARVANITOYANNIS, I. S.; KRYSTALLIS, A.; PANAGIOTAKI, P.; THEODOROU, A. J. A marketing survey on Greek consumers' attitudes towards fish. Aquaculture International, Amsterdã, v. 12, n. 3, p. 259-279, 2004.

BARBOSA, J. A.; SANTANA, A. C.; SILVA, I. M.; BOTELHO, M. N.; NETO, J. M. H. C. Características comportamentais do consumidor de peixe no mercado de Belém. Boletim Técnico Científico CEPNOR, Belém, v. 7, n. 1, p. 115-133, 2006.

BECKER, T. Consumer perception of fresh meat quality: a framework for analysis. British Food Journal, Reino Unido, v. 102, n. 3, p. 158-176, 2000.

BIRCH, D.; LAWLEY, M. Buying seafood. Understanding barriers to purchase across consumption segments. Food Quality and Preference, Amsterdã, v. 26, n. 1, p. 12-21, 2012.

BORTOLINI, G. A.; GUBERT, M. B.; SANTOS, L. M. P. S. Consumo alimentar entre crianças brasileiras com idade de 6 a 59 meses. Cadernos de Saúde Pública, Rio de Janeiro, v. 28, p. 1759-1771, 2012. 
BRUNSØ, K.; VERBEKE, W.; OLSEN, S. O.; JEPPESEN, L. F. Motives, barriers and quality evaluation in fish consumption situations: Exploring and comparin heavy and light users in Span and Belgium. British Food Journal, Reino Unido, v. 111, n. 7, p. 699-716, 2009.

CARLUCCI, D.; NOCELlA, G.; DEVITIIS, B. D.; VISCECCHIA, R.; BIMBO, F.; NARDONE, G. Consumer purchasing behaviour towards fish and seafood products. Patterns and insights from a sample of international studies. Appetite, Reino Unido, v. 84, p. 212-227, 2015.

COTIA INFORMAÇÕES E CONSULTAS. Centro de atendimento ao turista. Disponível em: http://www. prefeituradecotia.com.br/centro-de-atendimento-aoturista-em-cotia/. Acesso em: 05 mar. 2019.

CUNHA, C. F.; SPERS, E. E.; ZYLBERSZTAJN, D. Percepção sobre atributos de sustentabilidade em um varejo supermercadista. RAE-Revista de Administração de Empresas, São Paulo, v. 51, n. 6, p. 542-552, 2011.

EMATER. Empresa de Assistência Técnica e Extensão Rural. 2018. Disponível em: https://www.agrolink. com.br/culturas/peixes/noticia/produtores-de-peixe-sepreparam-para-a-semana-santa_405069.html. Acesso em: 10 jul. 2019.

FOOD AND AGRICULTURE ORGANIZATION OF TH UNITED NATIONS. The estate of world fisheries and aquaculture 2018 - Meeting the sustainable development goals. Roma: FAO, 2018.

FIGUEIRO, R. C.; SOUSA, J. M.; CASTRO, E. M. Fatores que influenciam na decisão de compra de pescado no mercado de peixe de Bragança-PA. Revista Brasileira de Engenharia de Pesca, Maranhão, v. 7, n. 1, p. 60-72, 2014.

GONÇALVES, A. A. Tecnologia do pescado: ciência, tecnologia, inovação e legislação. São Paulo, SP: Atheneu, 2011.

HAIR JUNIOR, J. F.; ANDERSON, R. E.; TATHAM, R. L.; BLACK, W. C. Análise multivariada de dados. Bookman: Porto Alegre, 2005.

HAWKINS, D.; MOTHERSBAUGH, D.; BEST, R. Comportamento do consumidor: Construindo a estratégia de marketing. Rio de Janeiro, RJ: Campus, 2017.
INSTITUTO BRASILEIRO DE GEOGRAFÍA E ESTATÍSTICA. Pesquisa de Orçamentos Familiares 2017 e 2018: Primeiros resultados. Rio de Janeiro, RJ: IBGE, 2019.

INFOPESCA. Centro para los servicios de información y asesoramiento sobre la comercialización de los produtos pesqueiros de América Latina y el Caribe. $\mathbf{O}$ mercado do pescado nas grandes cidades latino americanas. Santos, SP: INFOPESCA, 2010.

KAIMAKOUDI, E.; POLYMEROS, K.; SCHINARAKI, M. G.; BATZIOS, C. Consumers' Attitudes Towards Fisheries Products. Procedia Technology, Amsterdã, v. 8, p. 90-96, 2013.

KAWARAZUKA, N.; BÉNÉ, C. Linking small-scale fisheries and aquaculture to household nutritional security: an overview. Food Security, Berlim, v. 2, n. 4, p. 343-357, 2010. doi: 10.1007/s12571-010-0079-y

KHAN, A. Q.; ALDOSARI, F.; HUSSAIN, S. M. Fish consumption behavior and fish farming attitude in Kingdom of Saudi Arabia (KSA). Journal of the Saudi Society of Agricultural Sciences, Riade, v. 17, p. 195-199, 2016.

KOTLER, P.; ARMSTRONG, G. Princípios de marketing. São Paulo, SP: Pearson Education do Brasil, 2015.

LEEK, S.; MADDOCK, S.; FOXALL, G. Situational determinants of fish consumption. British Food Journal, Reino Unido, v. 12, p. 1, p. 18-39, 2000.

LOPES, I. G.; OLIVEIRA, R. G.; RAMOS, F. M. Perfil do consumo de peixes pela população brasileira. Biota Amazônia, v. 6, p. 62-65, 2016.

MAZZUCHETTI, R. N.;BATALHA, M. O. O comportamento do consumidor em relação ao consumo e às estruturas de comercialização da carne bovina na região de Amerios/PR. Revista Varia Scientia, Cascavel, v. 4, n. 8, p. 25-43, 2005.

MAlHOTRA, N. Pesquisa de marketing: uma orientação aplicada. Porto Alegre: Bookman, 2001.

MYRLAND, O.; TRONDSEN, T.; JOHNSTON, R. S.; LUND, E. Determinants of seafood consumption in Norway: lifestyle, revealed preferences, and barriers to consumption. Food Quality and Preference, Barking, v. 11, p. 169-188, 2000. 
MINOZZO, M. G.; HARACEMIV, S. M. C.; WASZCZYNSKJI, N. Perfil dos consumidores de pescado nas cidades de São Paulo (SP), Toledo (PR) e Curitiba (PR) no Brasil. Revista Alimentação Humana, Campinas, v. 14, n. 3, p.133-140, 2008.

NIELSEN, N. A.; SØRENSEN, E.; GRUNERT, K. G. Consumer motives for buying fresh or frozen plaice: a means end chain approach. IN: Grunert, K. G. (2005). Food quality and safety: consumer perception and demanda. European Review of Agricultural Economics, Oxford, v. 32, n. 3, p. 369-391, 1997.

OLSEN, S. O. Understanding the relationship between age and seafood consumption: the mediating role of attitude, health involvement and convenience. Food Quality and Preference, v. 14, p. 199-209, 2003.

PARENTE, J. Varejo no Brasil: Gestão e estratégia. São Paulo, SP: Atlas, 2010.

PEIXE-BR. Associação brasileira da piscicultura. Anuário Peixe BR da Piscicultura 2019. São Paulo/SP: Peixe BR, 2019.

PINHEIRO, R. M.; CASTRO, G. C.; SILVA, H. H. C.; NUNES, J. M. G. Comportamento do consumidor. Rio de Janeiro, RJ: FGV, 2015.

ROCHA, C. M. C.; RESENDE, E. K.; ROUTLEDGE, E. A. B.; LUNDSTEDT, L. M. Avanços na pesquisa e no desenvolvimento da aquicultura brasileira. Pesquisa Agropecuária Brasileira, Brasília, v. 48, n. 8, p. 4-6, 2013.

São Paulo Antiga. Conheça o Brooklin, o bairro que combina o melhor da modernidade e do conforto. 2019. Disponível em: http://www.saopauloantiga.com.br/ conheca-o-brooklin/. Acesso em: 15 jul. 2019.

SARTORI, A. G. O.; AMANCIO, R. D. Pescado: importância nutricional e consumo no Brasil. Segurança Alimentar e Nutricional, Campinas, v. 19, n. 2, p. 8393, 2012.

SCHARF, E. R. A proposta de valor e o capital humano: Práticas estratégicas de marketing. Revista Brasileira de Gestão de Negócios, São Paulo, v. 14, n. 43, p. 216233, 2012.

SOLOMON, M. Comportamento do consumidor: comprando, possuindo e sendo. Porto Alegre, RS: Bookman, 2016.

SONODA, D. Y.; SHIROTA, R. Consumo de pescado no Brasil fica abaixo da média internacional. Visão Agrícola, v. 11, p. 145-147, 2012.

SOUKI, G. Q.; SALAZAR, G. T.; ANTONIALLI, L. M.; PEREIRA, C. A. Atributos que afetam a decisão de compra dos consumidores de carne bovina. Revista de Administração da UFLA, v. 5, n. 2, p. 36-51, 2003.

SOUZA, M. L. R.. Comparação de seis métodos de filetagem, em relação ao rendimento de filé e de subprodutos do processamento da Tilápia-do-Nilo (Oreochromis niloticus). Revista Brasileira de Zootecnia, v. 31, n. 3, p. 1076-1084, 2002.

SUN, Y. H. C. Health concern, food choice motives, and attitudes toward healthy eating: the mediating role of food choice motives. Appetite, v. 51, n. 1, p. 42-49, 2008.

SUPARTINI, A.; OISHI, T.; YAGI, N. Changes in fish consumption desire and its factors: A comparison between the United Kingdom and Singapore. Foods, v. 7, n. 97, p. 1-9, 2018.

TAVARES, G. C.; AQUINO, R. M. A.; PALHARES, M. M.; SANTOS, R. R. D.; BONFIM, M. L.; TEIXEIRA, L. V. Perfil do consumo de pescado na cidade de Belo Horizonte, MG. Boletim de Indústria Animal, v. 70, n. 3, p. 230-236, 2013.

VASCONCELOS, Juliana Parreira. Determinantes do consumo de pescado na população que frequenta feiras livres do município de Santo André, SP. 2010. Dissertação (Mestrado em Epidemiologia Experimental Aplicada às Zoonoses, Universidade de São Paulo, São Paulo, 2010.

VERBEKE, W.; VACKIER, I. Individual determinants of fish consumption: application of the theory of planned behavior. Appetite, v. 44, p. 67-82, 2005. 